\title{
Apoyando la participación real de las personas con discapacidad intelectual: una experiencia de investigación inclusiva sobre vida independiente
}

\author{
Supporting the real participation of people with intellectual disability: \\ an inclusive research experience about independent living
}

\begin{abstract}
Resumen
En nuestro contexto se han desarrollado escasas experiencias de investigación realizadas conjuntamente entre investigadores académicos e investigadores con discapacidad intelectual. Este artículo tiene como finalidad aportar una experiencia de investigación cualitativa sobre vida independiente y discapacidad, desarrollada por las autoras (investigadoras académicas) en colaboración con un Consejo Asesor integrado por personas con discapacidad intelectual. Se pretende, además de presentar el desarrollo y resultados de la investigación, explicar cómo se ha construido el proceso de colaboración entre los asesores y las investigadoras de la universidad a lo largo de las distintas fases del estudio. También se van a concretar las estrategias de apoyo que se han ofrecido a los asesores para plantear, finalmente, las reflexiones sobre los principales retos que conlleva avanzar en los procesos de investigación inclusiva. Con ello se pretende contribuir al avance de las investigaciones inclusivas en las que las personas con discapacidad tengan un protagonismo real en el estudio de los problemas que las afectan.
\end{abstract}

\section{Palabras clave}

Investigación inclusiva, discapacidad intelectual, vida independiente, investigación colaborativa.

\begin{abstract}
In our context, few studies have been developed jointly between academic researchers and researchers with intellectual disabilities. The aim of this article is to describe a qualitative research experience about independent living and intellectual disabilities conducted by the authors (academic researchers), in collaboration with an Advisory Committee composed of people with intellectual disabilities. Aside from exposing the development and the results of the investigation, this article aims at narrating the collaboration process between the advisors and the university researchers along the different research phases. The support strategies offered to the advisors are also detailed, as well as some considerations about the main challenges faced along inclusive researches. This paper intends to contribute to the advancement of inclusive research, capable of ensuring that people with disabilities have a real role in the study of the problems that affect them.
\end{abstract}

\section{Keywords}

Inclusive research, intellectual disabilities, independent living, collaborative research.
María Pallisera Díaz

<maria.pallisera@udg.edu>

Instituto de Investigación Educativa, Universidad de Girona

Judit Fullana Noell
<judit.fullana@udg.edu>

Instituto de Investigación Educativa, Universidad de Girona

\section{Carol Puyaltó Rovira} <carolina.puyalto@udg.edu>

Instituto de Investigación Educativa, Universidad de Girona

\section{Montserrat Vilà Suñé}

<montserrat.vila@udg.edu>

Instituto de Investigación Educativa, Universidad de Girona

\section{Gemma Díaz Garolera <gemma.diaz@udg.edu>}

Instituto de Investigación Educativa, Universidad de Girona

Para citar:

Pallisera, M. et al. (20I7): "Apoyando la participación real de las personas con discapacidad intelectual: una experiencia de investigación inclusiva sobre vida independiente". Revista Española de Discapacidad, 5 (I): 7-24.

Doi: <https://doi.org/IO.5569/23405104.05.0I.OI>

Fecha de recepción: I6-I I-20I6 Fecha de aceptación: 09-05-20I7 


\section{Introducción}

La Convención sobre los Derechos de las Personas con Discapacidad (ONU, 2006) supone no solo el reconocimiento de las personas con discapacidad como sujeto de derecho, sino también la necesidad de orientar los procesos de investigación a la promoción de los derechos de estas personas y a sus necesidades reales (García-Iriarte et al., 20I6). Desde esta perspectiva, la participación de las personas con discapacidad intelectual en las investigaciones sobre temas que las afectan es necesaria para focalizar la investigación en aquellas cuestiones que realmente les importan y que repercuten de forma significativa en sus trayectorias personales (Johnson, 2009). Así, la Convención refuerza la necesidad de potenciar investigaciones en las que las personas con discapacidad intelectual tienen una participación activa y que, desde finales de los 90 (especialmente en el contexto anglosajón), se han ido realizando de forma creciente configurando el denominado modelo de investigación inclusiva (Walmsley, 200I, 2004; Walmsley y Johnson, 2003). La incorporación de las experiencias y opiniones de las personas con discapacidad intelectual en la investigación enriquece tanto el proceso como los resultados del estudio y aumenta las posibilidades de incidir de forma significativa en la mejora de sus condiciones de vida a través de la defensa de sus derechos (Johnson et al., 20I4; O'Brien et al., 20I4). Desarrollar los procesos de investigación de manera que las personas que han sido hasta este momento focos pasivos de estudio jueguen un papel activo en las distintas fases de la investigación conlleva, además, cuestionar el propio proceso desde una óptica que entienda que su conocimiento es valioso, necesario e importante para la creación de conocimiento $\mathrm{y}$, en consecuencia, replantear el papel de los investigadores académicos como sujetos que controlan el proceso (Parrilla y Sierra, 20I 5 ).

Aunque en los últimos I 5 años se ha publicado un número creciente de investigaciones llevadas a cabo de forma inclusiva con personas con discapacidad intelectual, en la mayoría de los casos no se aporta información sobre qué apoyos se les ha ofrecido ni cómo se han desarrollado las acciones de apoyo (Bigby y Frawley, 20Io; Tuffrey-Wijne y Butler, 2009). A menudo tampoco se detallan cuáles han sido las responsabilidades de las personas con discapacidad a lo largo de la investigación. La transparencia de los procesos de investigación inclusiva es necesaria no solo para facilitar su evaluación, sino también para aportar conocimientos, aprendizajes y orientaciones a la comunidad científica (Nind y Vinha, 20I4). Por ello, algunos investigadores insisten en la necesidad de que en este tipo de investigaciones se concrete "quién ha hecho qué" (Walmsley, 2004; Nind et al., 201 5); es decir, explicar cuál ha sido la aportación de investigadores con y sin discapacidad a lo largo del proceso de investigación.

En nuestro contexto, a pesar de las voces que reclaman potenciar investigaciones inclusivas (González Luna, 20I3; Haya et al., 20I4; Susinos y Parrilla, 2008 y 20I3; Parrilla y Sierra, 20I 5), la participación de las personas en situación vulnerable, como las personas con discapacidad intelectual en los procesos de investigación, es aún muy escasa. Por ello, se hace todavía más necesario difundir y analizar tanto resultados como procesos de investigación desarrollados de forma inclusiva, profundizando en los apoyos implementados y debatiendo las estrategias que pueden facilitar la generalización de experiencias de investigación inclusiva.

Partiendo de estas bases, este artículo tiene como finalidad aportar una experiencia de investigación desarrollada por las autoras (investigadoras académicas), en colaboración con un Consejo Asesor (CA) integrado por personas con discapacidad intelectual. Además de presentar el desarrollo y resultados de la investigación, se explica cómo se ha construido el proceso de colaboración entre los asesores y las investigadoras de la universidad a lo largo de la investigación. También se concretan las estrategias de apoyo que se han ofrecido a los asesores y se plantean, en último término, algunas reflexiones sobre los principales retos que conlleva avanzar en los procesos 
colaborativos con personas con discapacidad en la investigación sobre los temas que las afectan.

El primer apartado del artículo se centra en las aportaciones sobre los apoyos necesarios que deben implementarse en el contexto de investigaciones inclusivas para facilitar la construcción de procesos de trabajo conjuntos entre investigadores con y sin discapacidad. A continuación se presenta la investigación, centrada en la vida independiente y desarrollada por las autoras en colaboración con el Consejo Asesor, detallando las acciones de apoyo llevadas a cabo. Finalmente, en el último apartado se aportan las reflexiones y propuestas de mejora a partir de esta experiencia.

2. ¿Cómo apoyar procesos de investigación colaborativa con personas con discapacidad intelectual? Aportaciones a partir de investigaciones inclusivas.

El desarrollo de investigaciones desde planteamientos inclusivos ha generado gran número de debates acerca de distintas temáticas, entre las cuales destacan la cuestión de la representatividad de las personas con discapacidad intelectual en las distintas fases de la investigación (Bigby et al., 20I4), la equidad en las relaciones entre investigadores con y sin discapacidad (o el difícil equilibrio de poder) (Barton, 2005; Dowse, 2009), y el apoyo a los investigadores con discapacidad para participar activamente en las distintas fases del proceso (Bigby y Frawley, 20I0; Strnadová et al., 2014; Walmsley, 2004). En este artículo nos centramos concretamente en este último tema. Por ello, en este apartado se recogen las orientaciones que aportan distintos investigadores a partir de su experiencia en la realización de investigaciones en colaboración con investigadores con discapacidad intelectual. Se revisan las siguientes cuestiones: la construcción del equipo de investigación, la formación en investigación para los investigadores con discapacidad, el análisis de la información y la difusión de los resultados de la investigación.
La construcción del equipo de investigación es un tema que apenas se ha abordado en el análisis de investigaciones inclusivas. Sin embargo, se trata de una cuestión fundamental puesto que la cohesión del equipo es condición necesaria para el éxito del proceso y de los propios resultados de la investigación (Strnadová et al., 20I4). De hecho, algunas de las investigaciones centradas en las perspectivas y opiniones de los investigadores con discapacidad resaltan la importancia que tiene para ellos sentir que forman parte de un grupo que trabaja con un objetivo común y en el que sus voces son importantes (The Money, Friends and Making Ends Meet Research Group y Tilly, 20I2; Nind y Vinha, 20I4; Puyalto et al. 2016). Ello implica la necesidad de tener en cuenta, en las decisiones sobre el tipo de reuniones, su duración, la temporalización y la propia dinámica de las reuniones, que los integrantes del grupo se sientan progresivamente miembros de un equipo de personas que trabajan conjuntamente para conseguir un objetivo común (Pallisera et al., 2015 ). Como señala Johnson (2009), ello será particularmente importante cuando los miembros del grupo no tienen una trayectoria de trabajo en común (al contrario de lo que ocurre en los grupos de autogestión (self-advocacy groups), colectivos integrados por personas con discapacidad intelectual que discuten y defienden conjuntamente sus propios derechos.

Para contribuir a la construcción del equipo de investigación, planificar y desarrollar acciones de formación sobre investigación dirigidas a los investigadores con discapacidad puede constituir una estrategia potente. La necesidad de formación en investigación es un tema reconocido tanto por investigadores sin discapacidad (Bigby y Frawley, 2010; Johnson, 2009; Pallisera y Puyalto, 20I4; Puyalto et al., 20I6; Strnadová et al., 20I4; Walmsley, 2004; Walmsley y Johnson, 2003; Ward y Simons, I998) como por las propias personas con discapacidad que han participado en investigaciones llevadas a cabo de forma inclusiva (Abell et al., 2007; Burke et al., 2003; Butler et al. 20I2; Flood et al., 20I 2; White y Morgan, 20I2). Como plantean Nind et al. (2OI5) la cuestión de la formación no es 
neutra, puesto que implica plantear cual es el objetivo de la formación: ¿̇se trata de facilitar el aprendizaje de competencias relacionadas con la investigación para que los investigadores con discapacidad puedan abordar tareas más complejas a lo largo del proceso?, ¿o bien contribuir a proporcionar a las personas con discapacidad un rol y un estatus socialmente valorado como colaboradores en procesos rigurosos de contribución a la creación de conocimiento?

La revisión de la literatura ofrece pocas referencias acerca de la orientación de los procesos formativos desarrollados con personas con discapacidad para capacitarles en competencias de investigación. Entre las recomendaciones derivadas de estos procesos se aconseja incidir en la comprensión del concepto de investigación y las cuestiones a las que el equipo quiere responder (Strnadová et al., 20I4). Se sugiere también plantear suficientes actividades prácticas para aplicar los conocimientos (Abell et al., 2007; Flood et al., 2012) y utilizar materiales accesibles para los participantes (Flood et al., 201 2; Burke et al., 2003; Salmon et al., 2014; Carey et al., 20I4). Además, se recomienda incluir en la formación cuestiones éticas relacionadas con la investigación (Abell et al., 2007; Burke et al., 2003) y que los participantes puedan interactuar con grupos de personas con discapacidad que realizan investigaciones colaborativas para compartir experiencias (Flood et al., 20I2). Johnson (2009), en el proyecto "No longer researching about us without us", insiste en las posibilidades del role playing y las prácticas de observación. Salmon et al. (20I4) y Carey et al. (20I4) desarrollan y evalúan el Research Active Programme (RAP), indicando la necesidad de individualizar las acciones formativas de acuerdo con las necesidades de los participantes, implicando en la formación a estudiantes de profesiones relacionadas con la atención a personas con discapacidad intelectual $\mathrm{u}$ otros profesionales, además de facilitar que los participantes puedan evaluar cada sesión.

El desarrollo de una investigación implica distintos procesos: el planteamiento del tema y las cuestiones de investigación, la planificación del proceso, la recogida de información e incluso el análisis de los datos obtenidos. En la investigación con personas con discapacidad el apoyo a estos procesos implica facilitar su participación real y garantizar la accesibilidad a la información.

El análisis de la información es un proceso transversal que se realiza en distintos momentos de una investigación con distintas finalidades: al inicio de (y durante) la investigación, para recabar datos sobre el tema de estudio; una vez recogidos los datos, para profundizar en su significado y establecer relaciones con los resultados de otras investigaciones. Y una vez finalizada la investigación, para seleccionar y organizar la información necesaria para la difusión de los resultados.

Sin duda, se trata de una de las dimensiones más complejas, por su diversidad y trascendencia, del proceso de investigación. Algunos autores (Nind, 20I I) señalan que probablemente no todos los aspectos del análisis de datos sean accesibles a los investigadores con discapacidad. Sin embargo, según esta autora, las personas con discapacidad podrían participar conjuntamente en la interpretación de los datos y los resultados de la investigación, contribuyendo de este modo al avance del conocimiento. Stevenson (20I4) muestra que los co-investigadores pueden adquirir habilidades de análisis de datos relacionadas con la codificación y la identificación de temas a partir de los datos, con cierto nivel de estructuración y complejidad, si se les proporciona la formación adecuada. En el trabajo de Tuffrey-Wijne y Butler (2009) se describe con detalle el proceso de colaboración establecido por ambos autores (el segundo con discapacidad intelectual) en la fase de análisis de datos cualitativos procedentes de una investigación etnográfica, y se aportan sus reflexiones sobre las posibilidades que puede brindar el apoyo de los investigadores académicos en esta fase de la investigación.

O’Brien et al. (20I4) señalan la necesidad de que los investigadores con discapacidad dispongan de conocimiento sobre la temática de 
análisis y sobre las investigaciones anteriores, que debe ser mostrada de forma accesible; ello puede realizarse mediante diversas estrategias como, por ejemplo, invitando a personas con experiencia en la temática de estudio, presentando resultados de informes en formato accesible, o bien discutiendo de forma abierta (por ejemplo, a través de brainstorming) la importancia del tópico.

Finalizada la investigación, diseminar los resultados es una fase imprescindible para garantizar su posible impacto en las políticas y las prácticas. O'Brien et al. (2OI4) subrayan la necesidad de que los investigadores de la universidad o las propias organizaciones de las personas con discapacidad inviertan esfuerzos para permitir la difusión de los resultados y con ello su incidencia en la mejora de las prácticas y las políticas. Ahora bien, la equidad se configura como un reto cuando nos enfrentamos a la difusión de los resultados desde una perspectiva colaborativa, tanto por la complejidad de las tareas que implican las formas tradicionales de difusión dirigidas a la comunidad científica y profesional, basadas en narrativa escrita, como por las limitadas vías que admiten formatos accesibles. Son escasas las publicaciones científicas de carácter periódico que, en estos momentos, admiten artículos accesibles (lenguaje sencillo, acompañado de ilustraciones) dirigidos a la comunidad científica o a la ciudadanía. Solo la revista The British Journal of Learning Disabilities ha publicado algunos artículos accesibles en cuya redacción han participado personas con discapacidad. En cuanto a congresos, los organizados por la Asociación Internacional para el Estudio Científico de las Discapacidades Intelectuales y del Desarrollo (IASSID) exigen resúmenes accesibles y permiten presentar comunicaciones accesibles. En los dos casos, se trata de vías de difusión de difícil acceso para los investigadores con discapacidad intelectual. Esta situación pone de relieve la necesidad de buscar canales de difusión alternativos en los que los investigadores con discapacidad puedan participar.

\section{3. "Dónde quiero vivir y con quién": acciones de apoyo implementadas para potenciar la participación de los asesores con discapacidad en la investigación}

El artículo i 9 de la Convención sobre los Derechos de las Personas con Discapacidad (2006) establece el derecho de todas las personas con discapacidad a decidir dónde quieren vivir y con quién quieren hacerlo. También dice que éstas tienen derecho a recibir apoyos para participar en la comunidad. Aun así, la investigación demuestra que las personas con discapacidad intelectual se enfrentan a muchos impedimentos para ejercer este derecho (Inclusion International, 20I 2; FRA-European Union Agency for Fundamental Rights, 20I2). En España predomina la atención residencial en el contexto institucional: la mayoría de las personas con discapacidad viven en residencias $\mathrm{u}$ hogares de grupo como alternativas a la vivienda familiar y apenas se han desarrollado alternativas de servicios basados en la comunidad (Verdugo et al., 2009). Tal como plantea la Convención sobre los Derechos de las Personas con Discapacidad, es necesario estudiar en profundidad las dificultades y los apoyos que se encuentran las personas con discapacidad intelectual para vivir según sus deseos (art. 3I) y para que se tengan en cuenta sus experiencias y opiniones (art. 33). En este contexto se lleva a cabo la investigación "Dónde vivir y con quien” en colaboración entre el equipo de investigación y el Consejo Asesor, con el objetivo de profundizar en las barreras y los apoyos que experimentan las personas con discapacidad intelectual para su vida independiente.

En el siguiente apartado se describe, paralelamente al proceso de investigación seguido, las acciones de apoyo llevadas a cabo por las investigadoras de la universidad en los distintos momentos de la investigación. Concretamente, nos referimos a las estrategias desarrolladas para potenciar la construcción del grupo, las relacionadas con la formación y el acceso a la información en distintas fases de la investigación (documentación acerca de 
la temática de estudio, obtención y análisis de datos y discusión de los mismos), y la difusión de los resultados.

\subsection{Acciones planteadas para facilitar la construcción y cohesión del grupo}

Esta investigación se llevó a cabo conjuntamente entre un grupo de investigadoras de la Universidad de Girona y un Comité Asesor integrado por personas con discapacidad intelectual que colabora con el grupo desde el curso 2012-I3. En 2012 el grupo de investigación empezó un estudio sobre el proceso de transición de jóvenes con discapacidad intelectual a la vida adulta ${ }^{\mathrm{I}}$. Queríamos que la investigación explicara los problemas reales a los que se enfrentan las personas con discapacidad en este proceso. Por ello consideramos necesario incorporar en la investigación un Consejo Asesor integrado por personas con discapacidad intelectual, una de las vías para realizar investigaciones inclusivas (Bigby et al., 20I4; Walmsley y Johnson; 2003), con el objeto de que sus miembros asesoraran, a partir de sus experiencias personales, las distintas fases del proceso de investigación.

Durante el primer año de funcionamiento se realizaron seis reuniones a razón de una por mes (Pallisera et al., 20I 5). El reto principal fue cohesionar tanto el grupo de asesores, procedentes de cinco servicios distintos, como establecer una dinámica de trabajo conjunta entre el Consejo Asesor y el equipo de investigadoras que fuera tanto efectiva como enriquecedora a nivel del proceso de colaboración. Las acciones llevadas a cabo para ayudar a establecer las sinergias necesarias para construir un equipo de trabajo fueron las siguientes:

I. Esta investigación fue financiada por el MINECO (Gobierno de España) a través del proyecto EDU20II-22945 (La transición a la edad adulta y vida activa de jóvenes con discapacidad intelectual desde una perspectiva inclusiva: identificación de problemas, buenas prácticas y propuesta de un plan de mejora).
- Utilización del método del grupo focal en las reuniones, valorando entre sus fortalezas el hecho de que configura un entorno seguro para la discusión que ayuda a los participantes a ganar confianza, a colaborar y a recibir apoyo del resto del grupo, y a contrastar opiniones y experiencias (Cambridge y McCarthy, 200I; Barr et al., 2003; Kaehne y O’Connell, 2010). Además, permite que las personas con pocas habilidades de lectoescritura puedan participar activamente en procesos de investigación.

- Creación de un espacio informal al inicio de cada reunión dedicado al intercambio de impresiones y a realizar un pequeño tentempié.

- Revisión conjunta de la reunión al finalizar cada sesión.

- Preparación cuidadosa de las reuniones teniendo en cuenta el desarrollo de la reunión anterior y la necesidad de utilizar materiales de apoyo accesibles (tanto las presentaciones como los documentos). Se redactó un acta accesible de cada reunión que se entregaba y comentaba a los participantes al inicio de la siguiente.

La experiencia de colaboración fue valorada por todos sus miembros muy positivamente (Puyalto et al., 2016), por lo que se decidió continuar con la colaboración. Así, el curso 20I3-I4, segundo año de funcionamiento del Consejo Asesor, propusimos a los asesores realizar conjuntamente una investigación sobre vida independiente y éstos aceptaron. Durante este segundo año se llevaron a cabo las acciones de formación en investigación para los asesores.

\subsection{La formación de los investigadores con discapacidad}

La formación consistió en un curso sobre investigación educativa organizado con un doble objetivo: por una parte, se pretendía facilitar a los participantes un mayor conocimiento 
del sentido y el alcance de los procesos de investigación como vía para contribuir al avance de conocimiento; por otro, se quería facilitar a los asesores un mayor conocimiento acerca de los procesos, instrumentos y estrategias básicas que utilizamos en nuestro equipo, de manera que pudieran valorar su disponibilidad y motivación para participar más activamente en la investigación. La formación se desarrolló a lo largo de ocho sesiones de trabajo y abarcó distintos temas: el sentido de la investigación en el ámbito socioeducativo, las fases de la investigación (desde la concreción del problema y la formulación de cuestiones de investigación a la selección, diseño y aplicación de instrumentos y análisis de datos, discusión de resultados y difusión) y, concretamente, se incidió en dos de las técnicas más empleadas por nuestro equipo: la entrevista y los grupos focales. También se profundizó en el uso de métodos visuales como dibujo y fotografía (Pallisera et al., 20I4). En nuestro caso, la formación se realizó simultáneamente al desarrollo de la investigación. Ello permitió aplicar algunos de los temas del curso a la propia investigación sobre vida independiente.

En las distintas sesiones participaron diferentes investigadores como formadores y se combinaron explicaciones con actividades prácticas que tenían como objetivo facilitar a los participantes la aplicación de los conocimientos aportados por el formador. Desde el equipo de investigación se ofreció apoyo tanto a los formadores (antes de la sesión, para contextualizar y orientar la elaboración de material accesible, y durante la sesión, para ayudar a dinamizarla) como a los participantes. Se buscó elaborar material docente con un fuerte componente visual para facilitar la comprensión de la información aportada. También se relacionaron las actividades formativas con la temática de la investigación que se estaba desarrollando sobre vida independiente.

\subsection{El acceso a la información}

En este apartado nos referimos a los apoyos implementados por parte de las investigadoras de la universidad en distintas fases de la investigación.

\subsubsection{Documentación sobre la temática de estudio}

Tal como puede observarse en la Tabla I, se realizaron tres sesiones dedicadas a obtener información acerca de los modelos actuales de apoyo a la vida independiente para personas con discapacidad intelectual. En esta fase de la investigación el apoyo de las investigadoras de la universidad se concretó en las siguientes acciones:

- Organización de una discusión abierta para obtener información sobre los conocimientos previos de los asesores y asegurar que el tema era suficientemente motivador para ellos (primera sesión).

- Exploración conjunta de las características, posibilidades y limitaciones de los distintos modelos de apoyo a la vida independiente en nuestro contexto utilizando material audiovisual (segunda sesión). En concreto, se seleccionaron fragmentos audiovisuales que muestran escenas donde interaccionan personas con discapacidad y profesionales en una residencia (Flynn y Wilson, 2004), en un hogar tutelado y en un hogar propio donde la persona recibe apoyo de forma puntual (Televisió de Catalunya, 2013).

- Realización de una sesión dedicada a discutir casos prácticos que mostraban situaciones próximas a partir de las cuales se valoraron las distintas variables implicadas (la voluntad de la persona, el papel de la familia, del empleo...) (tercera sesión). 


\begin{tabular}{|c|c|c|}
\hline Sesión & Objetivo & Desarrollo \\
\hline 1 & $\begin{array}{l}\text { Compartir conocimientos } \\
\text { previos acerca de los apoyos a } \\
\text { la vida independiente (VI). }\end{array}$ & $\begin{array}{l}\text { Se planteó una discusión abierta en el que cada asesor explicó } \\
\text { sus experiencias e ideas acerca de la vida independiente. Se } \\
\text { valoró que la persona debe poder escoger la que sea mejor para } \\
\text { ella entre las distintas opciones (vivir con los padres, en un hogar } \\
\text { con apoyo, etc.), así como la necesidad de disponer de recursos } \\
\text { económicos y de saberlos administrar. }\end{array}$ \\
\hline 2 & $\begin{array}{l}\text { Profundizar en las distintas } \\
\text { opciones de apoyo a la VI: } \\
\text { residencia, hogar tutelado, } \\
\text { apoyo personalizado en el } \\
\text { propio hogar. }\end{array}$ & $\begin{array}{l}\text { Se visionaron fragmentos de películas y documentales que } \\
\text { muestran las distintas opciones. A continuación se discutieron } \\
\text { los pros y contras de las distintas opciones. Los asesores } \\
\text { estuvieron de acuerdo en: } \\
\text { - Las personas deben poder escoger dónde vivir y con quién. } \\
\text { - Los profesionales deben potenciar siempre la autonomía de } \\
\text { las personas. } \\
\text { - La opción de recibir apoyo en el propio hogar. } \\
\text { - La educación familiar es muy importante para potenciar la } \\
\text { autonomía. }\end{array}$ \\
\hline 3 & $\begin{array}{l}\text { Valorar cómo las distintas } \\
\text { opciones de apoyo a la VI se } \\
\text { pueden adaptar a situaciones } \\
\text { personales concretas. }\end{array}$ & $\begin{array}{l}\text { Se discutió en pequeños grupos sobre diferentes casos prácticos } \\
\text { centrados en personas con discapacidad intelectual (DI) que } \\
\text { desean avanzar en su proceso de emancipación. Una de ellas } \\
\text { vivía en su casa, otra en un hogar tutelado. Los asesores } \\
\text { discutieron la situación personal valorando los deseos de los } \\
\text { protagonistas de los casos y planteando en cada uno de ellos las } \\
\text { opciones posibles. }\end{array}$ \\
\hline
\end{tabular}

Fuente: elaboración propia.

\subsubsection{Obtención y análisis de datos}

En esta investigación los asesores discutieron conjuntamente, con el apoyo de distintos métodos y a lo largo de varias sesiones, sobre cuáles son las principales barreras que se encuentran para conseguir sus proyectos de vida independiente. También valoraron los apoyos que les ayudan a avanzar en sus proyectos y formularon propuestas dirigidas a potenciar su vida independiente. Se realizaron concretamente dos sesiones, cuya descripción se muestra en la Tabla 2. Se ofreció previamente formación metodológica a los asesores sobre métodos visuales y desarrollo de grupos focales. Por una parte, un investigador académico experto en el uso de métodos visuales impartió en el curso de formación una sesión centrada en las posibilidades de la fotografía y el dibujo como apoyo a la narrativa oral. Por otra, dos investigadoras explicaron a los asesores las posibilidades y cuestiones técnicas relacionadas con el empleo de grupos focales y el apoyo a sus participantes. 


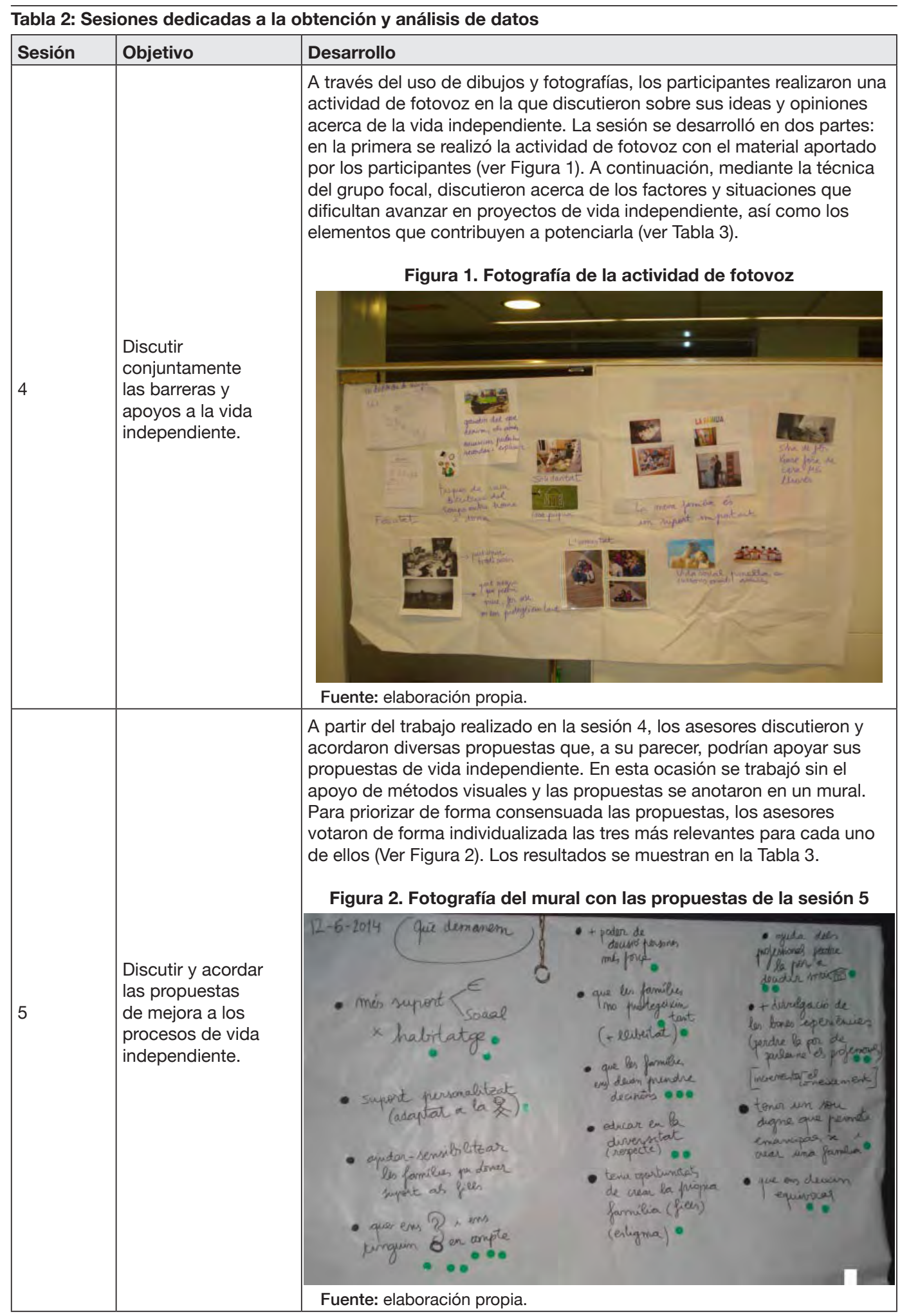

Fuente: elaboración propia. 


\begin{tabular}{|c|c|}
\hline \multicolumn{2}{|l|}{ Tabla 3: Resultados } \\
\hline Barreras & $\begin{array}{l}\text { - Falta de confianza de la gente en nuestras posibilidades. } \\
\text { - } \text { - Hacer frente a las rutinas del día a día. } \\
\text { - Miedo a emanciparse. } \\
\text { - Falta de ayudas económicas y de apoyo para vivir de forma } \\
\text { independiente. } \\
\text { - A veces, los profesionales no nos ayudan de forma adecuada. } \\
\text { - Demasiado control. }\end{array}$ \\
\hline Apoyos & $\begin{array}{l}\text { - Libertad para escoger y pedir. } \\
\text { - } \text { - Reciboyo y acompañamiento por parte de las familias. } \\
\text { independientes. } \\
\text { - Apoyo en el trabajo. } \\
\text { - Tener dinero suficiente. } \\
\text { - Tener amigos. } \\
\text { - Tener pareja. } \\
\text { - Tener la posibilidad de tomar decisiones. }\end{array}$ \\
\hline Propuestas de mejora & $\begin{array}{l}\text { - } \text { Que nos escuchen y nos tengan en cuenta. } \\
\text { - El gobierno debería apoyarnos para vivir de forma independiente, } \\
\text { - } \text { facilitándonos las viviendas y ayudándonos a pagarlas. } \\
\text { - } \text { decisiones. } \\
\text { - } \text { Eue los profesionales nos ayuden a perder el miedo a tomar decisiones. } \\
\text { - Que nos dejen equivocarnos. } \\
\text { - Difundir buenas experiencias. Que los profesionales no tengan miedo a } \\
\text { - } \text { hablar de ello. } \\
\text { - Tecibir apoyo personalizado. } \\
\text { - Tener la oportunidad de crear nuestra propia familia. }\end{array}$ \\
\hline
\end{tabular}

Fuente: elaboración propia.

La Tabla 3 resume los resultados de las valoraciones de los asesores en cuanto a barreras, apoyos y propuestas de mejora. A modo de ejemplo, añadimos algunas valoraciones en relación a los profesionales y a las familias:

"Los profesionales tienen que estar cualificados y deben permitir que los demás hagamos cosas porque, si no, no aprenderán nunca a hacerlas" (Asesor 2).

"Los padres sobreprotegen mucho. Pueden ponerte problemas cuando te quieres emancipar" (Asesor 4).

"Es importante que te estimulen desde pequeño, que no te malcríen. Es muy importante la educación que han dado los padres" (Asesor 3).

\subsubsection{Discusión de los resultados}

Contrastar los resultados propios con los de otras investigaciones que aborden la misma cuestión de estudio es una de las fases de la investigación que conlleva, por su complejidad, ciertas dificultades para los investigadores con discapacidad. Sin embargo, realizar este proceso de forma colaborativa puede contribuir a que éstos avancen en la comprensión global del sentido de una investigación y en valorar su contribución al avance del conocimiento. Se dedicó una sesión a trabajar conjuntamente la discusión de los resultados de la investigación desarrollada con los asesores (ver Tabla 4). Antes de la sesión, las investigadoras de la universidad revisaron diversas investigaciones que abordan las mismas cuestiones de investigación (barreras y apoyos en el proceso 
de VI y propuestas de mejora) y en las que, al igual que en nuestra investigación, son las propias personas con discapacidad quienes exponen sus opiniones acerca de estos temas. Se seleccionaron concretamente las siguientes fuentes: McConkey et al., 2004; Inclusive Research Network, 20I0; FRA, 20I2; McGlaughlin et al., 2004; Deguara et al., 20I2; Inclusion International, 2OI2. Se agruparon las aportaciones de los artículos en tres grupos: barreras, apoyos y propuestas de mejora. A continuación, se preparó una tarjeta con el texto de cada una de las aportaciones y sus autores (ver un ejemplo en la Figura 3).

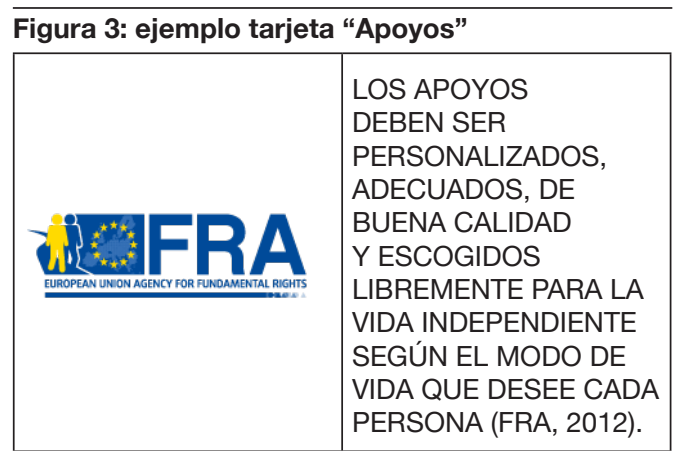

Fuente: elaboración propia.

En esta fase de la investigación, el apoyo de las investigadoras de la universidad se concretó en las siguientes acciones.

Los asesores se organizaron en tres grupos, cada uno de ellos contó con el apoyo de una de las investigadoras. A cada grupo se le facilitó el siguiente material:

- Un tablero magnético con imanes.

- Tarjetas con las aportaciones de otras investigaciones. Cada grupo se centró en un solo tema: apoyos, barreras, o propuestas de mejora.

- $\quad$ El listado de las aportaciones de la propia investigación.

Después de explicar en qué consistía esta fase de trabajo, se procedió a trabajar en grupo en la lectura y análisis de los textos para contrastar la información. Los asesores, con el apoyo de las investigadoras de la universidad, relacionaron las tarjetas con las aportaciones surgidas a partir del propio estudio. A continuación, se realizó una puesta en común en la que se comentaron los resultados y se valoró el procedimiento utilizado. Las investigadoras se encargaron de redactar, con el resultado del trabajo de los distintos grupos, la discusión de los resultados.

Tabla 4: Discusión de los resultados
\begin{tabular}{|l|l|l|}
\hline Sesión & Objetivo & Desarrollo \\
\hline \multirow{6}{*}{6} & $\begin{array}{l}\text { Comparar los } \\
\text { resultados de } \\
\text { nuestro estudio } \\
\text { con los de otras } \\
\text { investigaciones } \\
\text { que muestran } \\
\text { las opiniones de } \\
\text { personas con } \\
\text { discapacidad } \\
\text { sobre la VI. }\end{array}$ & $\begin{array}{l}\text { Los asesores se } \\
\text { grupos, cada uno de } \\
\text { los cuales comparó los } \\
\text { resultados del propio } \\
\text { estudio con los de otros } \\
\text { estudios previos. Un } \\
\text { grupo se centraba en } \\
\text { comparar las barreras, } \\
\text { otro comparaba los } \\
\text { apoyos y el tercero } \\
\text { se centraba en las } \\
\text { propuestas de mejora. }\end{array}$ \\
\hline
\end{tabular}

Fuente: elaboración propia.

\subsubsection{La difusión}

Desde el equipo de investigadoras se valoró la necesidad de difundir la experiencia, tanto su desarrollo como los resultados. Ello es imprescindible tanto para poder incidir en el avance de los planteamientos de apoyo a la vida independiente como para potenciar el reconocimiento de las personas con discapacidad intelectual como investigadoras y, con ello, contribuir a su reconocimiento y valorización social. La necesidad de difusión y de participar también colaborativamente en algunas de las acciones de difusión, se acordó conjuntamente con los asesores.

Concretamente se realizaron las siguientes actividades de difusión:

- Participación en jornadas, seminarios y cursos de verano: los asesores, junto 
con las investigadoras, explicaron la investigación en un curso de verano (julio de 20I4) y en una jornada sobre investigación colaborativa (mayo de 2015). Los destinatarios fueron profesionales, estudiantes e investigadores. En ambas ocasiones, las investigadoras preparamos el borrador de la presentación. Partiendo de éste, tanto asesores como investigadoras acordamos la estructura final, las imágenes que queríamos utilizar y los fragmentos a comentar. Las dos presentaciones se llevaron a cabo de manera conjunta.

- Colaboración en la página web del grupo de investigación. Durante una sesión realizada en enero del año 20 I $_{5}$, los asesores elaboraron, con el apoyo de las investigadoras, una ficha de presentación en la que se explica quiénes somos, qué hacemos y el resumen de nuestras experiencias desde el año $20 \mathbf{I}^{2}$.

- Elaboración de un póster para un congreso internacional. En abril de $20 \mathrm{I} 5$ preparamos un póster describiendo la investigación sobre vida independiente. Las investigadoras elaboramos una propuesta con la organización de los contenidos y, juntamente con los asesores, revisamos los textos del póster para determinar si eran suficientemente claros y comprensibles. También escogimos las imágenes que queríamos incluir. Este póster se presentó en un congreso internacional (Fullana et al., 20I 5 ).

- Elaboración de un informe accesible sobre la investigación. El equipo de investigación propuso a los asesores elaborar conjuntamente, fruto de la investigación, un informe accesible para ser publicado con posterioridad en la página web del grupo de investigación y ser difundido a servicios y profesionales. A partir de la ficha de presentación, del póster y de las presentaciones del curso de verano y de las jornadas, las investigadoras prepararon un primer borrador del artículo. Asesores e

2. Disponible en: http://media.wix.com/ugd/fe33eo_2322cioe 567542a8a 5e2 I I4c8729f 5ce.pdf. investigadoras discutieron conjuntamente el borrador, acordando los contenidos, la estructura y las imágenes (Coll et al., 20I6).

\section{Discusión y conclusiones}

"Podemos aportar muchas cosas de la propia experiencia... Si están hablando de la discapacidad, ¿qué mejores profesores que nosotros mismos?" (Asesor I).

Asumir que el conocimiento que aportan las personas con discapacidad intelectual acerca de su propia experiencia es imprescindible para mejorar sus condiciones de vida, avanzando hacia su inclusión social, implica transformar radicalmente los modos de hacer investigación que han dominado en este ámbito.

Desarrollar investigaciones inclusivas supone replantear las cuestiones metodológicas para favorecer la participación de las personas que pueden mostrar dificultades para acceder a las formas de conocimiento relacionadas con el proceso de investigación, pero también repensar el propio proceso de investigación teniendo en cuenta que todos los participantes son fuente de conocimiento que, aunque diverso, es igualmente valioso (Parrilla y Sierra, 20I 5 ). En este sentido, en el desarrollo de investigaciones inclusivas la experiencia y conocimiento de los investigadores académicos tiene un papel clave para diseñar y organizar de forma creativa los apoyos necesarios que permitan a colectivos vulnerables sentirse parte fundamental de la investigación y poder participar de forma activa en las distintas fases de la misma. Acciones como la construcción y cohesión del grupo de investigación y asegurar la accesibilidad a las diferentes fases de la investigación se configuran como requisitos necesarios para facilitar el desarrollo de investigaciones desde una óptica inclusiva.

Este artículo se ha elaborado con la voluntad de compartir una experiencia de investigación 
desarrollada de forma colaborativa entre investigadoras académicas y un grupo de personas con discapacidad intelectual. La experiencia descrita en este artículo permite discutir distintos temas vinculados con la realización de investigaciones inclusivas y con el apoyo a los investigadores con discapacidad intelectual para facilitar su participación en éstas.

La construcción del grupo de investigación supone un reto y está enormemente condicionada por la relación y experiencia previa de trabajo conjunto que tienen los participantes. En el caso de los asesores que han participado en la investigación sobre vida independiente, no tenían experiencia conjunta y apenas se conocían entre ellos, puesto que procedían de cinco servicios distintos. Tal como señala Johnson (2009), esta situación lleva a invertir necesariamente mayores esfuerzos a nivel de gestión, puesto que es necesario multiplicar los contactos con profesionales de los servicios de procedencia para negociar la participación de personas con discapacidad en la investigación. Implica, asimismo, plantear decididamente las estrategias necesarias para establecer sinergias de trabajo conjunto.

Aunque en nuestro caso algunos de los asesores formaban parte, a su vez, de grupos de autogestión, ésta no era la situación de la mayoría de los participantes. Ninguno de los participantes había participado anteriormente en procesos de investigación, por lo que desde el grupo de investigación se valoró la necesidad de realizar una formación orientada a conocer el sentido de la investigación, sus fases y algunas de las técnicas utilizadas por el grupo.

En nuestro caso, la formación en investigación se ha desarrollado en dos fases: en un formato tradicional (en forma de paquete o curso formativo) y a través de la participación colaborativa en una investigación sobre vida independiente. Ello se planteó con el convencimiento de que, sin duda, la formación ayuda pero no es suficiente para a aprender a ser investigador. Construir capacidades para investigar pasa por participar activamente al menos en algunas fases de la investigación.
La investigación colaborativa supone producir conocimiento a través de un proceso de reflexión conjunta. Aprender a desarrollar investigaciones colaborativas supone un reto puesto que implica que investigadores con y sin discapacidad aprendan juntos reconociendo que ambos aportan su conocimiento a la investigación pero que también necesitan el apoyo del resto del equipo (Walmsley, 2004; Nind et al., 20I 5 ). En la presente investigación los asesores procedían de distintos servicios y no tenían una experiencia de trabajo conjunto, por lo que la experiencia les ha permitido, como en la de Johnson (2009), discutir sobre sus vidas en un espacio diferente al que ocupan como usuarios de servicios; han podido establecer nuevos contactos en un nuevo entorno, así como nuevas relaciones, y han construido colectivamente un nuevo rol como investigadores. Con ello, han podido disponer de más elementos y más precisos para comprender las situaciones que viven, para poderlas analizar y, en definitiva, para poder emprender actuaciones que mejoren su propio contexto.

En la valoración realizada por los asesores acerca de su participación en la experiencia al finalizar la investigación, consideran que ésta ha tenido impacto en ellos mismos, en su relación con el entorno (familia, amigos) y también en su posicionamiento en relación a la defensa de sus derechos. En cuanto a los aprendizajes personales, valoran que su participación en la investigación les ha sido útil para aprender más sobre la sociedad, para tener mayor confianza en el grupo, para aprender cosas necesarias sobre el futuro y para mejorar su autoestima por el hecho de saber que sus opiniones son valoradas. Respecto a las relaciones con el entorno, se ven dotados de más instrumentos para poder hablar de temas sociales. Sus amigos y familiares se interesan más por lo que hacen y por sus opiniones y valoran el trabajo que realizan en la universidad. Y hacen nuevas amistades.

A partir de la experiencia desarrollada defendemos, como plantean O'Brien et al. (20I4), que no solo los participantes con discapacidad intelectual aprenden nuevas habilidades en su participación en investigaciones inclusivas; también los 
investigadores académicos hemos adquirido nuevas competencias relacionadas con la escucha de los colaboradores con discapacidad, con la organización y presentación de la información de forma accesible y con la adaptación continua de las actividades de la investigación a una dinámica que favorezca la participación activa y colaborativa de todos los participantes. Hemos aprendido a diseñar e implementar nuevas estrategias facilitadoras de la accesibilidad de la información que podemos extrapolar a la docencia y a otras actividades de investigación. Hemos hecho nuevas amistades y profundizado de forma significativa en la realidad de las personas con las que trabajamos e investigamos.

Más que la formación 'formalmente' establecida, es la propia participación en procesos de investigación lo que puede contribuir al empoderamiento de los participantes con discapacidad y, en este sentido, los grupos de investigación tienen un papel fundamental para incentivar el desarrollo de investigaciones inclusivas como estrategia dirigida a empoderar a las personas con discapacidad intelectual en la defensa de sus derechos, incidiendo en la transformación de las políticas y de las prácticas que les afectan (Fullana et al., 20I6).

Tal como Johnson (2009) señala, no todas las personas con discapacidad van a desear participar en procesos de investigación. En nuestro caso, algunos de los asesores que participaron en la investigación decidieron, durante el curso siguiente, dejar temporalmente el Consejo Asesor. En ello influyó la dificultad para compatibilizar esta participación con otras actividades lúdicas o laborales. También incidió el hecho de que la actividad de investigación del grupo se realizaba aparte de la dedicación laboral de los asesores, requiriendo una dedicación que algunos de ellos no disponían. Poder contar con recursos para reconocer la dedicación de los asesores con discapacidad a la investigación sería posiblemente un elemento que apoyaría la constitución de grupos de investigación con mayor estabilidad temporal. Otro elemento a tener en cuenta para potenciar la constitución de comunidades de investigación estables es que los resultados de las investigaciones desarrolladas inclusivamente tengan una incidencia real en la mejora de las condiciones de vida de los participantes (García Iriarte et al., 20I4). En este sentido, la difusión de los resultados es fundamental, aunque los canales mayoritariamente académicos establecidos para este fin pongan barreras a la participación de las personas con discapacidad en la difusión. En nuestro caso se han buscado alternativas accesibles como la página web o la elaboración de informes accesibles. Sin embargo, deberían crearse mecanismos que permitieran una mayor participación de las personas con discapacidad en la difusión de los procesos y resultados de la investigación.

En la investigación presentada la temática fue seleccionada por las investigadoras académicas. Aunque los asesores aceptaron participar en ella y se mostraron a lo largo de todo el proceso interesados en profundizar en las distintas cuestiones, ellos no participaron en la determinación el tema. En un contexto en el que la financiación va dirigida fundamentalmente a grupos de investigación reconocidos, y en el que los grupos de autogestión tienen aún una escasa trayectoria y no han incorporado la investigación como tarea propia, deben dedicarse esfuerzos conscientemente dirigidos a explicar a estos grupos la necesidad de la investigación como estrategia de mejora de sus condiciones de vida, y a potenciar su implicación en ellos. Además, tal como plantean O’Brien et al. (2OI4), la constitución de comunidades de investigación estables que puedan optar a obtener financiación para estudiar y mejorar aquello que les interesa constituye un reto al que deben dirigirse los esfuerzos necesarios para avanzar en el equilibrio de las relaciones de poder entre los investigadores con y sin discapacidad. En este sentido, la voluntad y el compromiso de los investigadores académicos en democratizar los procesos de investigación, cediendo el control que han asumido tradicionalmente en ellos, es fundamental puesto que juegan un papel clave en la dinamización y apoyo de investigaciones inclusivas. Aún así, como plantean Goodlay y Moore (2010), debemos ser conscientes de que la capacidad 
de los investigadores para transformar las condiciones de producción del conocimiento mediante la investigación son limitadas; la democratización de los procesos de investigación exige modificaciones significativas de las formas tradicionales de difusión para poder incluir de forma real y efectiva a las personas con discapacidad intelectual en los procesos de diseminación.

La investigación inclusiva no es solo una potente fuente de conocimiento sobre las realidades de los colectivos vulnerables, sino también un instrumento de transformación en si mismo. Es un instrumento efectivo para contribuir al cambio social puesto que tiene en cuenta las necesidades y peticiones reales de las personas, y permite que sean ellas mismas quienes provoquen esta transformación. Ahora bien, para que esta transformación pueda ser realmente efectiva, deben plantearse más oportunidades tanto para que las personas con discapacidad participen activamente en las investigaciones que las afectan como para que puedan jugar un papel protagonista en la difusión de las investigaciones realizadas. La universidad tiene como reto el convertirse en un espacio realmente inclusivo en el que la participación de colectivos vulnerables como las personas con discapacidad intelectual no sea excepcional en la investigación y diseminación de sus resultados, potenciando el acceso de la ciudadanía tanto a la producción de conocimiento como a sus resultados. Para ello, deben cuestionarse y revisarse a fondo las formas tradicionales de generar y difundir conocimiento vinculadas a la universidad, para establecer vías alternativas que contribuyan a democratizar los procesos de investigación, haciendo accesibles sus resultados al conjunto de la sociedad. 
Referencias bibliográficas

Abell, S. et al. (2007): "Including everyone in research: The Burton Street Research Group". British Journal of Learning Disabilities, 35: I2I-I 24 .

Barr, O. et al. (2003): "Views of People with Learning Difficulties about Current and Future Accommodation: The use of focus groups to promote discussion". Disability \& Society, I 8 (5): 577-597.

Barton, L. (2005): “Emancipatory research and disable people: some observations and questions”. Educational Review, 57 (3): 317327.

Bigby, C. et al. (20I4): "Conceptualizing Inclusive Research with People with Intellectual Disability". Journal of Applied Research in Intellectual Disabilities, 27: 3-I2.

Bigby, C. y Frawley, P. (2010): "Reflections on doing inclusive research in the 'Making Life Good in the Community' study". Journal of Intellectual Developmental Disability, 35 (2): 53-6r.

Burke, A. et al. (2003): "Setting up participatory research: a discussion of the initial stages". British Journal of Learning Disabilities, 3 I (2): 65-69.

Butler, G. et al. (20I 2): "Doing it together". British Journal of Learning Disabilities, 40: I34-I42.

Cambridge, P. y McCarthy, M. (200I): “User focus groups and Best Value in services for people with learning disabilities". Health and Social Care in the Community, 9: 476-489.

Carey, E. et al. (2014): “Service user's views of the Research Active Programme". Learning Disability Practice, I7 (4): 22-28.

Coll, T. et al. (2016): "On viure i amb qui". Barreres $i$ suports per a la vida independent des del punt de vista dels assessors amb discapacitat (en línea). <http://media.wix.com/ugd/fe 33 eo_ e36da8fere7947789ca32f 75 I 7443 fb3.pdf>, acceso ro de octubre de 2016.
Deguara, M. et al. (2012): "How we like to live when we have the chance". British Journal of Learning Disabilities, 40: I23-I 27.

Dowse, L. (2009): “It's like being in a zoo. Researching with people with intellectual disability". Journal of Research in Special Educational Needs, 9 (3): I4I-I 53.

Flood, S. et al. (2OI2): "Becoming a researcher". British Journal of Learning Disabilities, 4I: 288-295.

Flynn, J. y Wilson, J. (prods.) (2004): Inside I'm Dancing. [Película]. Irlanda.

FRA-European Union Agency for Fundamental Rights (2OI2): Choice and control: the right to independent living. Experiences of persons with intellectual disabilities and persons with mental health problems in nine EU Member States. Luxemburgo: Publications Office of the European Union.

Fullana, J. et al. (20I6): "Las personas con discapacidad intelectual como investigadoras. Debates, retos y posibilidades de la investigación inclusiva”. EMPIRIA. Revista de Metodología de Ciencias Sociales, 33: I I I-I 38.

Fullana, J. et al. (2015): "The path to the independent living: experiences, opinions and suggestions from people with intellectual disabilities in an inclusive research project". En rza Research Conference. Póster llevado a cabo en la conferencia de la Nordic Network of Disability Research, Bergen, Noruega (en línea). <http://media.wix.com/ugd/fe $33 \mathrm{eo}_{-}$ dfc 5 afd 206934 f9fad $92 a_{7} b_{4}$ edao7c5.pdf $>$, acceso 30 de septiembre de 2016.

Garcia-Iriarte, E. et al. (2016): Disability and Human Rights. Global perspectives. London: Palgrave.

García-Iriarte, E. et al. (20I4): "Involving people with intellectual disabilities within research teams: lessons learned from an Irish experience". Journal of Policy and Practice in Intellectual Disabilities, I I (2): I49-I 57. 
González Luna, B. (2013): "Investigando con personas con dificultades de aprendizaje". Revista Española de Discapacidad, I (2): 77-94.

Goodley, D. y Moore, M. (2010): “Doing Disability Research: Activist lives and the academy". Disability \& Society, I 5 (6): 86I882 .

Haya, I. et al. (2014): “Observaciones metodológicas sobre la investigación inclusiva: 'Me gustaría que sacarais que la persona con discapacidad tiene su propio pensamiento'”. Revista de investigación en educación, I 2 (2): I35-I 44 .

Inclusion International (20I 2): Inclusive Communities $=$ Stronger Communities: Global report on Article I9: the right to live and be included in the community. London: Inclusion International.

Inclusive Research Network (2010): Where we live: A national study done by members of the Inclusive Research Network through surveys. Dublin: National Federation of Voluntary Bodies.

Johnson, K. et al. (20I4): "Inclusive Research: Making a Difference to Policy and Legislation". Journal of Applied Research in Intellectual Disabilities, 27: 76-84.

Johnson, K. (2009): "No longer researching about us without us: a researcher's reflection on rights and inclusive research in Ireland". British Journal of Learning Disabilities, 37: 250-256.

Kaehne, A. y O’Connell, C. (20I0): "Focus groups with people with learning disabilities". Journal of Intellectual Disabilities, I4 (2): I33-I 45.

McConkey, R. et al. (2004): "Views of people with Intellectual Disabilities of Their Present and Future Living Arrangements". Journal of Policy and Practice in Intellectual disabilities, I (3-4): I I3-I 25 .

McGlaughlin, A. et al. (2004): "Enabling Adults with Learning Disabilities to Articulate their Housing Needs". British Journal of Social Work, 34 (5): 709-726.

Nind, M. et al. (2015): "The Conundrum of Training and Capacity Building for People with Learning Disabilities Doing Research”. Journal of Applied Research in Intellectual Disabilities, 29 (6): 542-55 I.

Nind, M. y Vinha, H. (20I4): "Doing research inclusively: bridges to multiple possibilities in inclusive research". British Journal of Learning Disabilities, 42 (2): I02-I09.

Nind, M. (20I I): "Participatory data analysis: a step too far?”. Qualitative Research, I I (4): 349-363.

O'Brien, P. et al. (20I4): “Co-researching with people who have intellectual disabilities: Insights from a National Survey". Journal of Applied Research in Intellectual Disabilities, 27: 65-75.

Organización de las Naciones Unidas, ONU (2006): Convención sobre los Derechos de las Personas con Discapacidad. New York: ONU.

Pallisera, M. et al. (20I 5): "Una experiencia de investigación inclusiva. Personas con discapacidad intelectual como asesoras en una investigación sobre transición a la edad adulta". Revista Iberoamericana de Educación, 69 (2): I 47-I 66.

Pallisera, M. y Puyalto, C. (20I4): "La voz de las personas con discapacidad intelectual en la investigación: hacia el desarrollo de investigaciones inclusivas". Revista Internacional y Nacional de Educación Inclusiva, 7 (2): 84-97.

Pallisera, M. et al. (20I4): "Investigamos junt@s: Un curso de formación en investigación educativa para personas con discapacidad intelectual”. En XI Congreso Internacional y XXXI Jornadas de Universidades y Educación Inclusiva. Comunicación llevada a cabo en el congreso de la Universitat Jaume I de Castellón, España.

Parrilla, A. y Sierra, S. (20 I 5): “Construyendo una investigación inclusive en torno a las distintas transiciones educativas". Revista Electrónica Interuniversitaria de Formación del Profesorado, I 8 (I): I6I-I75.

Puyalto, C. et al. (20I6): "Doing research together: a study on the views of advisors with intellectual disabilities and non-disabled 
researchers collaborating in research". Journal of Applied Research in Intellectual Disabilities, 29 (2): I46-I 59.

Salmon, N. et al. (2014): "Research Skills for People with Intellectual Disabilities". Learning Disability Practice, I 7 (3): 27-35.

Stevenson, M. (20I4): "Participatory Data Analysis alongside co-researchers who have Down Syndrome". Journal of Applied Research in Intellectual Disabilities, 27: 23-33.

Strnadová, I. et al. (20I4): "Building an Inclusive Research Team: The importance of team building and skills training”. Journal of Applied Research in Intellectual Disabilities, 27: I3-22.

Susinos, T. y Parrilla, A. (2013): "Investigación inclusiva en tiempos difíciles. Certezas provisionales y debates pendientes". Revista Electrónica Iberoamericana sobre Calidad, Eficacia y Cambio en Educación, I I (2): 88-98.

Susinos, T. y Parrilla, A. (2008): "Dar la voz en la investigación inclusiva. Debates sobre inclusión y exclusión desde un enfoque biográficonarrativo". Revista Electrónica Iberoamericana sobre Calidad, Eficacia y Cambio en Educación, 6 (2): I 57-I7I.

Televisió de Catalunya (prod.) (2013): El Convidat: Andy Trias [programa de televisión]. Barcelona: Televisió de Catalunya (en línea). <http://www. ccma.cat/tv3/alacarta/el-convidat/andy-trias/ video/4686834/>, acceso 30 septiembre 2016 .

The Money, Friends and Making Ends Meet Research Group y Tilly, L. (2OI2): “Having friends - they help you when you are stuck from money, friends and making ends meet research group". British Journal of Learning Disabilities, 40: I28-I33.

Tuffrey-Wijne, I. y Butler, G. (2009): “Coresearching with people with learning disabilities: an experience of involvement in qualitative data analysis". Health Expectations, I3: I74-I 84 .

Verdugo, M. A. et al. (2009): Country report on the implementation of policies supporting independent living for disabled people. España: Academic Network of European Disability Experts.

Walmsley, J. (2004): “Involving users with learning difficulties in Health improvement: lessons from inclusive learning disability research". Nursing Inquiry, I I: 54-64.

Walmsley, J. y Johnson, K. (2003): Inclusive Research with People with Learning Disabilities: Past, Present and Future. London: Jessica Kingsley Publishers.

Walmsley, J. (200I): “Normalisation, emancipatory research and inclusive research in learning disability". Disability \& Society, I6: I 87-205.

Ward, L. y Simons, K. (I998): "Practising Partnership: Involving People with Learning Difficulties in Research". British Journal of Learning Disabilities, 26: I 28-I 3 I.

White, E. L. y Morgan, M. F. (20I 2): “Yes! I am a researcher. The research story of a young adult with Down Syndrome". British Journal of Learning Disabilities, 40: IOI-Iо8. 